\title{
Erratum to: Laparoscopic ventral/incisional hernia repair: updated Consensus Development Conference based guidelines
}

\author{
Gianfranco Silecchia $^{1}$ - Fabio Cesare Campanile ${ }^{2}$ Luis Sanchez ${ }^{3}$. \\ Graziano Ceccarelli ${ }^{4}$ - Armando Antinori ${ }^{5} \cdot$ Luca Ansaloni $^{6} \cdot$ Stefano Olmi $^{7}$. \\ Giovanni Carlo Ferrari $^{8}$ - Diego Cuccurullo ${ }^{9}$ Paolo Baccari ${ }^{10} \cdot$ Ferdinando Agresta $^{11}$. \\ Nereo Vettoretto ${ }^{12} \cdot$ Micaela Piccoli $^{13}$
}

Published online: 13 August 2015

(C) Springer Science+Business Media New York 2015

\section{Erratum to: Surg Endosc \\ DOI 10.1007/s00464-015-4293-8}

The correct title is Laparoscopic ventral/incisional hernia repair: updated Consensus Development Conference based guidelines.

The online version of the original article can be found under doi:10.1007/s00464-015-4293-8.

\section{Fabio Cesare Campanile}

campanile@surgical.net

1 Division of General Surgery and Bariatric Centre of Excellence, Department of Medico-Surgical Sciences and Biotechnology, Sapienza University of Rome, Via Faggiana 1668, 04100 Latina, LT, Italy

2 Division of General Surgery, Ospedale San Giovanni Decollato Andosilla, ASL VT, Via Ferretti 169, 01033 Civita Castellana, VT, Italy

3 Division of Oncologic Surgery, Department of Oncology, Azienda Ospedaliero-Universitaria Careggi, Largo Brambilla 3, 50134 Florence, Italy

4 Division of General Surgery, Ospedale di Spoleto, Via Loreto 3, 06049 Spoleto, PG, Italy

5 Department of Surgery, Catholic University of Rome, Largo A. Gemelli 8, 00168 Rome, Italy

6 Division of General Surgery I, Papa Giovanni XXIII Hospital, Piazza OMS 1, 24127 Bergamo, Italy

7 Division of General and Oncologic Surgery, Advanced Laparoscopy Center, Policlinico San Marco, Zingonia, BG, Italy
8 Division of General, Oncologic and Mini-Invasive Surgery, A.O. Ospedale Niguarda Cà Granda, Piazza Ospedale Maggiore 3, 20162 Milan, Italy

9 Division of General, Laparoscopic and Robotic Surgery, AORN dei Colli Ospedale Monaldi, Naples, Italy

10 Division of General Surgery, Santa Maria della Misericordia Hospital, P.zale Menghini 1, 06129 Perugia, Italy

11 Department of General Surgery, ULSS19 del Veneto, Via Etruschi 9, 45011 Adria, RO, Italy

12 Laparoscopic Surgical Unit, M.Mellini Hospital, Viale Giuseppe Mazzini 4, 25032 Chiari, BS, Italy

13 Department of Surgery, NOCSAE (Nuovo Ospedale Civile Sant'Agostino Estense), Via Giardini 1355, 41126 Baggiovara, Modena, Italy 